\title{
Phylogeny and fitness of Vibrio fischeri from the light organs of Euprymna scolopes in two Oahu, Hawaii populations
}

\author{
Michael S Wollenberg and Edward G Ruby \\ Department of Medical Microbiology and Immunology, University of Wisconsin-Madison, Madison, WI, USA
}

\begin{abstract}
The evolutionary relationship among Vibrio fischeri isolates obtained from the light organs of Euprymna scolopes collected around Oahu, Hawaii, were examined in this study. Phylogenetic reconstructions based on a concatenation of fragments of four housekeeping loci (recA, $m d h, k a t A$, pyrC) identified one monophyletic group ('Group-A') of $V$. fischeri from Oahu. Group-A $V$. fischeri strains could also be identified by a single DNA fingerprint type. $V$. fischeri strains with this fingerprint type had been observed to be at a significantly higher abundance than other strains in the light organs of adult squid collected from Maunalua Bay, Oahu, in 2005. We hypothesized that these previous observations might be related to a growth/survival advantage of the Group-A strains in the Maunalua Bay environments. Competition experiments between Group-A strains and nonGroup-A strains demonstrated an advantage of the former in colonizing juvenile Maunalua Bay hosts. Growth and survival assays in Maunalua Bay seawater microcosms revealed a reduced fitness of Group-A strains relative to non-Group-A strains. From these results, we hypothesize that there may exist trade-offs between growth in the light organ and in seawater environments for local V. fischeri strains from Oahu. Alternatively, Group-A V. fischeri may represent an example of rapid, evolutionarily significant, specialization of a horizontally transmitted symbiont to a local host population.
\end{abstract}

The ISME Journal (2012) 6, 352-362; doi:10.1038/ismej.2011.92; published online 21 July 2011

Subject Category: microbe-microbe and microbe-host interactions

Keywords: biogeography; intraspecific; mutualism; phylogenetics; symbiosis

\section{Introduction}

The bioluminescent, heterotrophic marine bacterium Vibrio fischeri (Gammaproteobacteria: Vibrionaceae) maintains a mutualistic, binary relationship with bobtail squids of the genera Euprymna, Sepiola and Rondeletiola (Cephalopoda: Sepiolidae), and fishes of the genera Monocentris and Cleiodopus (Actinopterygii: Beryciformes). The host animals are hypothesized to receive a behavioral benefit from bacterial bioluminescence, whereas the bacteria are hypothesized to receive a metabolic benefit from the host (Stabb and Millikan, 2009). The Hawaiian bobtail squid, Euprymna scolopes, in particular, has been used for more than two decades as a model to study host-microbe interactions (McFall-Ngai and Ruby, 1991; Visick and Ruby, 2006). One characteristic of the squid/Vibrio model is the ease of laboratory cultivation of the host. Adult E. scolopes will mate in aquaria and lay clutches

Correspondence: EG Ruby, Department of Medical Microbiology and Immunology, University of Wisconsin-Madison, Madison, WI 53706-1521, USA.

E-mail: egruby@wisc.edu

Received 28 March 2011; revised 6 June 2011; accepted 6 June 2011; published online 21 July 2011 of eggs that produce hundreds of hatchlings. Another characteristic is the potential to manipulate symbiosis initiation in these newly hatched juveniles. $V$. fischeri are horizontally transmitted between squid generations and juvenile squid emerge from eggs uncolonized by $V$. fischeri (Wei and Young, 1989). As a result of this mode of transmission, V. fischeri populations are known to exist both in a specific organ in the host (the light organ) as well as the seawater environment (Lee and Ruby, 1992).

We recently analyzed the population structure of mutualistic $V$. fischeri collected from the light organs of adult E. scolopes from two populations on Oahu, Hawaii (Wollenberg and Ruby, 2009). Host light organs were found to contain polyclonal symbiont populations-population structure was assessed with a combination of physiological and genetic assays, including a novel $V$. fischeri DNAfingerprinting technique (VfRep-PCR). Interestingly, strains identified by one fingerprint type (VfRepPCR type-I) were found to be significantly more abundant than strains identified by other fingerprint types in the light organs of hosts collected from a specific environment (Maunalua Bay).

This former study was confined to describing patterns of $V$. fischeri population structure in the 
light organs of particular host populations. Here, we bridge the gap between the observation of a single, highly abundant fingerprint type in the Maunalua Bay host population and hypothesis testing of ideas about both the evolutionary history and the contemporary fitness of this group in different niches. The study described below consisted of two distinct, but complementary analyses. First, a phylogenetic scheme was used to reconstruct the evolutionary relationship among $V$. fischeri strains with distinct VfRep-PCR types. Second, different fitness assays were performed on representatives of these types in two Maunalua Bay environments: the squid host and natural seawater. The phylogenetic scheme and fitness assays were used to test the following hypotheses:

(i) Strains with distinct VfRep-PCR types also form distinct (and/or monophyletic) clades within an evolutionary reconstruction of $V$. fischeri, and

(ii) Strains identified as VfRep-PCR type-I are more fit in both the Maunalua Bay host and seawater environments than other VfRep-PCR types.

\section{Materials and methods}

V. fischeri strains

$V$. fischeri strains were selected for use in this study to satisfy two goals: (1) The strains represent a diverse group of VfRep-PCR types based on our previous analysis (Wollenberg and Ruby, 2009) of symbiont population diversity on Oahu; and (2) the strains represent an ecologically, temporally and geographically broad sampling of $V$. fischeri. A core group of $45 \mathrm{~V}$. fischeri strains was selected from our laboratory collection (Figure 1 and Supplementary Table S1). Two closely related outgroup strains,
V. salmonicida LFI1238 and V. logei SA6, were used to root phylogenetic reconstructions and provide an outgroup when required by common population genetics statistics.

\section{Molecular phylogenetic analyses}

A multi-locus sequence analysis scheme was used to organize the molecular data for the strains used in this study. A previously published $V$. fischeri scheme (Mandel et al., 2009) that included three housekeeping loci (recA, mdh and katA) was expanded by adding one additional locus (pyrC); two loci involved with bioluminescence (ainS, $\operatorname{lux} A$ ) were analyzed for comparative purposes. New sequences from this analysis have been submitted to the GenBank database under accession numbers JF509753-JF509942. Specific information on the multi-locus sequence analysis scheme is summarized in the Supplementary Information and Supplementary Table S2.

Multiple sequence alignments for each collection of locus fragments were generated by using the MUSCLE (Edgar, 2004) algorithm as implemented in SeaView 4.2.8 (Gouy et al., 2010). Similarly to previous descriptions (Mandel et al., 2009; Bose et al., 2011), phylogenetic reconstructions assuming a tree-like topology were created by using three common methods: maximum parsimony; maximum likelihood (ML) and Bayesian inference (Bayes). Specifics of these methods, as well as descriptive analyses of molecular sequence data, are detailed in the Supplementary Information and Supplementary Table S3.

Phylogenetic reconstructions not assuming a treelike topology were conducted by using the programs SplitsTree 4.10 (Huson and Bryant, 2006) to highlight and clarify inconclusive recombination results,

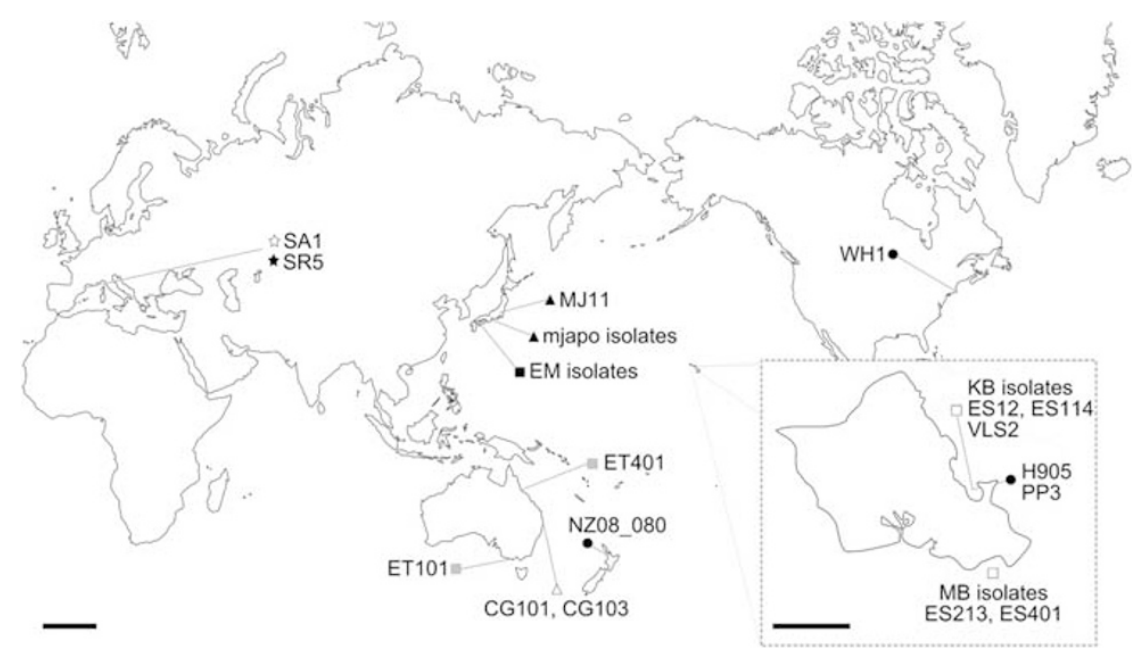

Figure 1 Geographic distribution of the $V$. fischeri strains used in this study. The majority of these strains were sampled from locations in the Pacific Ocean, specifically the Hawaiian Island of Oahu (inset). The shapes next to strain names denote the strains' ecological habitat: Filled black circle, planktonic (seawater); triangle or square or star, light-organ symbiont. The triangle, square and star fill colors represent host organisms as follows: White triangle, C. gloriamarus; black triangle, $M$. japonicus; white square, E. scolopes; gray square, E. tasmanica; black square, E. morsei; white star, S. affinis; black star, S. robusta. The bars in the main figure and inset represent 2000 and $20 \mathrm{~km}$, respectively. The background continent and Oahu outlines were taken from maps made available freely at http://d-maps.com/. 
and ClonalFrame 1.1 (Didelot and Falush, 2007) to address conflicting signals among the different gene fragments. For each concatenated data set, three independent runs of ClonalFrame were performed; in each run, the $50 \%$ majority rule consensus genealogy was estimated from the posterior distribution of 200000 generations (thinning interval of 100) following a burn-in of 100000 generations. Markov Chain Monte Carlo convergence was judged to be satisfactory by using the Gelman-Rubin test (Gelman and Rubin, 1992). The resulting posterior distribution of 6000 samples (three runs of 2000 samples each) from each concatenated data set was analyzed by using a consensus network (Holland et al., 2004) with mean edge weights at a threshold of 0.2 and an equal-angle splits transformation as implemented in SplitsTree. Nodes with 95\% posterior probability (from the 6000 original ClonalFrame samples) were identified in each network graph.

\section{Juvenile squid experiments}

Adult E. scolopes were collected from Maunalua Bay (MB), Oahu, Hawaii, and transported to aquaria in Madison, Wisconsin. Matings between MB adults and subsequent egg laying, development and hatching took place in these same aquaria. Hatchlings from MB adult matings were immediately moved into bowls containing $50-200 \mathrm{ml}$ of SFTW $(0.2-\mu \mathrm{m}$ sterile-filtered tank water) taken from an aquarium. All aquarium water was made from Instant Ocean (Spectrum Brands, Madison, WI, USA). For each experiment, approximately equal concentrations of two $V$. fischeri strains from different VfRep-type groups were inoculated into bowls containing 50-200 ml of SFTW; dilutions of this mixture were spread on SWT (seawater-tryptone) agar plates (Boettcher and Ruby, 1990) to assess the initial concentrations of bacteria. Newly hatched juveniles $(n=5-20)$ were added to a bowl; after either 3 or $18 \mathrm{~h}$, the juveniles were moved to individual glass vials containing $3 \mathrm{ml}$ of fresh SFTW. The animals were kept in these vials for an additional $30-45 \mathrm{~h}$, with a water change at approximately $20 \mathrm{~h}$. Next, juvenile squid were anesthetized on wet ice, frozen at $-80{ }^{\circ} \mathrm{C}$ and individually resuspended and homogenized in SFTW. Dilutions from this homogenate were then spread on SWT agar plates for enumeration of the relative proportions of the two strains by using their distinctive yellow or white colony pigmentations (Wollenberg and Ruby, 2009). Each competition experiment was evaluated by using the logarithm of the relative competitive index (RCI) to the base 10 (Stabb and Ruby, 2003). If the expression $X_{Y}$ is defined as the concentration of bacterial strain $\mathrm{X}$ after time Y, the RCI of Strain-A versus B after $48 \mathrm{~h}$ in the host is defined by the following equation: $\mathrm{RCI}_{\mathrm{AB}}=\left[\left(\mathrm{A}_{48} / \mathrm{B}_{48}\right) /\left(\mathrm{A}_{0} / \mathrm{B}_{0}\right)\right]$.

For each single-strain colonization experiment, approximately 1000 colony-forming units (c.f.u.) $\mathrm{ml}^{-1}$ of a single $V$. fischeri strain were inoculated into a bowl containing SFTW. Approximately $2.5 \mathrm{ml}$ of this $\mathrm{SFTW} / V$. fischeri mixture were added to each of 45 separate glass scintillation vials. Forty-five newly hatched juveniles were added individually to each of the vials. The animals were kept in these vials for an additional $24-72 \mathrm{~h}$, with a water change at approximately $24 \mathrm{~h}$ and $48 \mathrm{~h}$. At $24 \mathrm{~h}, 48 \mathrm{~h}$ and $72 \mathrm{~h}$ after addition, 15 animals were anesthetized on wet ice for $5 \mathrm{~min}$, frozen, homogenized and plated on SWT agar as described above. Data were summarized by recording the c.f.u./squid for each successful colonization event at each time point (per 15 squid). Because we are more concerned with the means of these data, and less with the variation among the individual data points, we report 95\% confidence interval and s.e.m., rather than standard deviation. Single-strain colonization experiments were performed with six strains (ES213, ES114, MB11B1, KB2B1, MB14A3 and MB15A4) representing the three major Hawaiian clades found in phylogenetic reconstructions (Figure 2 and Supplementary Figure S2).

For all squid experiments, aposymbiotic juveniles were placed in SFTW as a negative control. Briefly, 5-10 newly hatched juvenile squid were placed in a

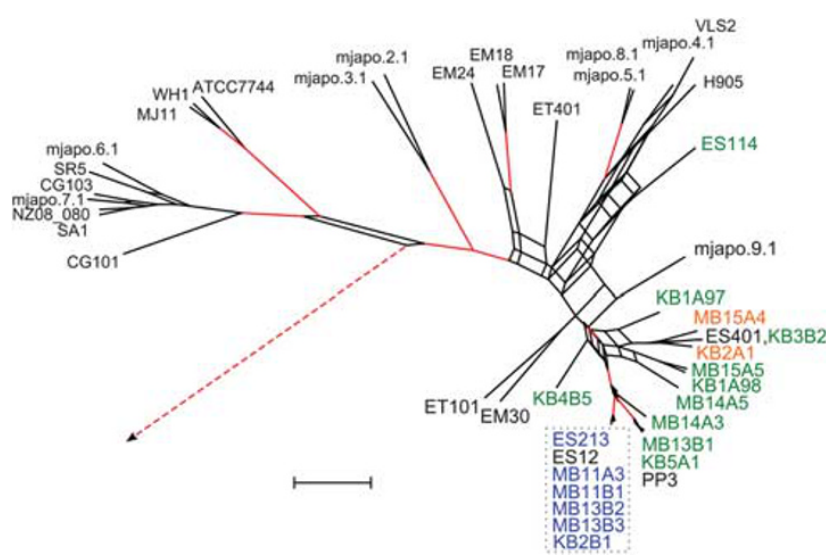

Figure 2 V. fischeri VfRep-PCR type-I strains form a monophyletic group within a phylogenetic reconstruction of global $V$. fischeri. A consensus network inferred from 6000 phylograms produced by ClonalFrame analyses of all taxa using a concatenation of four loci: recA, mdh, katA and pyrC. ClonalFrame was used to infer genealogical relationships from posterior distributions of 2000 samples from three independent runs $(3 \times 2000=6000$ total samples $)$. This aggregate ClonalFrame posterior distribution was used by SplitsTree to build a consensus network. For this network, reticulate relationships found in at least $20 \%$ of the sample may be visualized by parallel branches in parallelograms; nodes supported by a posterior probability greater than $95 \%$ (as summarized by ClonalFrame) are at the terminus of red-colored branches. The root (dashed red branch with arrow) has been truncated to expand the branches of the main $V$. fischeri group; the root contains both outgroup taxa: $V$. salmonicida LFI1238 and V. logei SA6. VfRep-PCR patterns have been mapped onto E. scolopes symbionts with colors: identically colored strains all share the same VfRep-PCR pattern (or type) (Supplementary Table S1): Blue, type-I (boxed); orange, type-II; green, type-III. The strains within the dotted box have been designated as 'Group-A'. The black bar is equivalent to a branch length of 0.1 substitutions per site. 
bowl containing only SFTW immediately after hatching. After $72 \mathrm{~h}$, the squid were sacrificed as above. In all experiments, none of these squid were colonized by more than 28 c.f.u.s of $V$. fischeri per squid (the lowest limit of detection assayed; data not shown).

\section{Hawaiian seawater experiments}

Six $V$. fischeri strains carrying the kanamycinresistant $\left(\operatorname{Kan}^{\mathrm{R}}\right)$, green fluorescent protein-expressing plasmid pVSV102 were constructed by triparental mating as described previously (Wollenberg and Ruby, 2009). These six strains (ES213, ES114, MB11B1, KB2B1, MB14A3 and MB15A4) represented the three major Hawaiian clades found in phylogenetic reconstructions (Figure 2 and Supplementary Figure S2). It was necessary to create $\mathrm{Kan}^{\mathrm{R}}$, green fluorescent protein strains because of the high background of naturally occurring, SWT-culturable bacteria in fresh MB seawater (see Supplementary Information for a more detailed description).

For growth experiments, the six labeled strains constructed above were individually grown in fresh, MB seawater-containing microcosms (Lee and Ruby, 1994). Briefly, a frozen stock culture of each strain was streaked onto three separate SWT agar plates containing $100 \mu \mathrm{g} \mathrm{ml}^{-1}$ kanamycin and grown for $24 \mathrm{~h}$ at $22-24{ }^{\circ} \mathrm{C}$. A colony scrape from each of these plates was diluted in $0.22-\mu \mathrm{m}$-filtered fresh $\mathrm{MB}$ seawater to a concentration of $\sim 10^{3}$ c.f.u. $\mathrm{ml}^{-1}$. These three (biological) replicates were performed for statistical purposes. Each diluted seawater culture was used as the primary inoculum of two experimental microcosms containing $3 \mathrm{ml}$ of either $0.22-\mu \mathrm{m}$-filtered or unfiltered $\mathrm{MB}$ seawater. The microcosms were shaken at $28{ }^{\circ} \mathrm{C}$ in an enclosed orbital shaker (New Brunswick Scientific, Edison, NJ, USA) at 225 r.p.m. for $96 \mathrm{~h}$ with periodic sampling and plating on SWT agar plates containing $100 \mu \mathrm{g} \mathrm{ml}^{-1}$ kanamycin. Colony abundance data were transformed into concentration data and analyzed by two-way, repeated-measures analysis of variance. The two factors of interest were the strains' group membership and time in hours (and their interaction). Correlation of repeated measures over time was accounted for by using random effects for strains (nested in groups) and for replicates (nested in strains). Analyses were conducted by using the statistical software R (R_Development_Core_Team, 2010) and the following statistical model: $\quad$ CFU $/ \mathrm{ml}=$ Group $*$ Hours + Error (Strain/Replicate). Statistical significance was evaluated with F-tests.

\section{Results}

Estimation of evolutionary parameters and ML gene phylogenies from individual locus fragments

We compared sequence alignment characteristics and ML-estimated evolutionary parameters for each of the six locus fragments from the $45 \mathrm{~V}$. fischeri strains analyzed in this study. The two locus fragments associated with quorum signaling and/or bioluminescence (ainS, luxA) contained nearly twice the percentage of variable and parsimonyinformative sites, per locus, when compared with the other four loci (Supplementary Table S3). For each locus fragment, the substitution parameters were complex, with four or more rate parameters as estimated by hierarchical likelihood ratio tests. Accounting for among-site rate variation significantly improved the model fit for each locus fragment $(P<0.001)$ (Supplementary Table S3).

Single-locus, ML phylogenetic reconstructions of all strains demonstrated that few general similarities or statistically significant nodes were common among tree topologies (Supplementary Figure S1). Notably, the reconstruction based on the $\operatorname{lux} A$ fragment contained nearly twice as many statistically supported nodes in comparison to each of the reconstructions based on the other five locus fragments. Upon inspection, this relatively high resolution could be explained by two factors: (i) 23 different $\operatorname{lux} A$ alleles exist among the strains, the second-lowest number of alleles among the six loci analyzed; and (ii) among these 23 alleles, the $\operatorname{lux} A$ fragments analyzed contain the lowest percentage of constant sites of any of the six loci analyzed. In other words, the $\operatorname{lux} A$ tree appears to have been well-resolved relative to the other five loci because its reconstruction was based on few alleles containing many nucleotide differences (Supplementary Table S3).

To further understand patterns of nucleotide polymorphism among these loci, more detailed test statistics were calculated (Supplementary Table S4). For all of these metrics, $\operatorname{lux} A$ stood out from the other five loci. First, the luxA locus fragment has a much larger number of total mutations than segregating sites, meaning that this locus generally has more than one polymorphism at each polymorphic site. Next, the average number of pairwise difference (k) and nucleotide diversity $(\pi)$ metrics have their highest values for luxA. Finally, estimations of both recombination and mutation parameters by various methods demonstrated that $\operatorname{lux} A$ has the lowest ratio of recombination rate to mutation rate $(\mathrm{c} / \mu)$ of all loci, reflecting both a low recombination parameter and a high mutation parameter relative to the other five loci (Supplementary Table S4). Together, these metrics indicate that, of the six locus fragments analyzed for these $V$. fischeri strains, the lux $A$ fragment has accumulated a relatively large number of polymorphisms through mutations, but has also been simultaneously constrained to a small number of absolute alleles. One hypothesis for this observation is that selection has been acting on $\operatorname{lux} A$ within the $V$. fischeri metapopulation. To evaluate this hypothesis, tests of the neutral equilibrium model of molecular evolution (Kimura, 1983) were conducted. 
When patterns of nucleotide substitutions for these six loci were analyzed under a neutral equilibrium model, this model was strongly rejected for $\operatorname{lux} A$ and weakly rejected for ainS (Table 1, Tajima's $D$ ). Because both selection and/or population demographics may explain statistically significant rejections of the null model for any single test (Tajima, 1989), multiple tests were employed to arrive at a clearer idea of the underlying processes responsible for the observed polymorphism in the data. Test statistics of Tajima's $D$ were significantly positive for both ainS and luxA, indicating an excess of polymorphisms relative to polymorphic sites, as implied by $\varnothing_{\pi}>\varnothing_{\mathrm{W}}$ (Table 1). A significantly positive value of $D$ is indicative of balancing selection on a particular locus and/or a population bottleneck. Tajima's $D$ was calculated for each sequence by using both nucleotide and amino-acid sequences-the latter, as noted (Vos and Velicer, 2006), may be more relevant in assessing evidence for selection because only non-synonymous polymorphisms are considered.

Other test statistics, such as Fu and Li's $D$ and $F$, Fu's $F$ s and Ramos-Onsis and Rozas's $R_{2}$ are more sensitive to population demographics than Tajima's $D$, and are often used to infer the role that demographic processes may have had in a given data set's nullmodel rejection (Simonsen et al., 1995). Using these tests, there was ample evidence that departure from neutral expectations by $\operatorname{lux} A$ may be due to demographic event(s); that is, these significantly large, positive test statistics imply that a population bottleneck may be responsible for the infrequency of polymorphic sites relative to the polymorphisms in the luxA data set. Interestingly, in the ainS data set, only $\mathrm{Fu}$ and Li's $F$ is significant, implying that demography may have less of a role in any significant violations of the neutral equilibrium model for this locus (Table 1).
Intragenic and intergenic recombination within and among loci

Evidence for intragenic recombination within each locus was analyzed by using a suite of algorithms included in the RDP software package; recombination events were only detected within the 783-bp mdh locus fragments. This result was mitigated by truncating the $3^{\prime}$-end of this fragment to remove the sequences showing evidence of intragenic recombination, resulting in a 519-bp $m d h$ locus fragment (data not shown). In addition, although there were no well-supported intragenic recombination events characterized for $k a t A$ and pyrC, these two fragments had a relatively high ratio of recombination rate to mutation rate (Supplementary Table S4), suggesting the presence of conflicting evidence both for and against the role of intragenic recombination within these loci.

The possibility of intergenic recombination among $V$. fischeri strains was qualitatively supported by the lack of similarity among nodes for each singlelocus ML reconstruction (Supplementary Figure S1). Topological incongruence among single-locus trees is one of the proposed hallmarks of intergenic recombination (Dykhuizen and Green, 1991). The hypothesis of topological incongruence among individual locusfragment reconstructions was found to be statistically well-supported and ubiquitous under both ML and maximum parsimony frameworks (Supplementary Table S5).

Another method for assessing evidence for intergenic recombination is the calculation of several different summary statistics of recombination, the most common being the standardized index of association $\left(I_{\mathrm{A}}^{S}\right)$ and the global ratio of recombination rate to point mutation rate for all loci $(r / m)$. For the six loci studied, the START2 program calculated $I_{\mathrm{A}}^{S}=0.3218$ and rejected the null hypothesis of linkage equilibrium $\quad\left(V_{\mathrm{O}}=0.7096, \quad V_{\mathrm{e}}=0.272\right.$,

Table 1 Selection and population growth test statistics from six loci of 45 different $V$. fischeri strains

\begin{tabular}{|c|c|c|c|c|c|c|c|c|}
\hline \multirow{2}{*}{$\begin{array}{l}\text { Locus } \\
\text { fragment }\end{array}$} & \multirow[t]{2}{*}{$\omega^{\mathrm{a}}$} & \multicolumn{3}{|c|}{ Tajima's $\mathrm{D}^{\mathrm{b}, \mathrm{c}}$} & \multirow[t]{2}{*}{ Fu and Li's $\mathrm{D}^{\mathrm{c,d}}$} & \multirow[t]{2}{*}{ Fu and Li's $\mathrm{F}^{\mathrm{c}, \mathrm{d}}$} & \multirow[t]{2}{*}{$F u{ }^{\prime} \mathrm{Fs}^{\mathrm{c}, \mathrm{d}}$} & \multirow[t]{2}{*}{$\mathrm{R}_{2}^{\mathrm{e}}$} \\
\hline & & S & $\eta$ & $\mathrm{AA}^{\mathrm{f}}$ & & & & \\
\hline $\operatorname{rec} A$ & $0.0122(0.0020,0.0376)$ & -0.744 & -0.860 & -1.475 & -1.201 & -1.311 & -3.578 & 0.083 \\
\hline$m d h$ & $0.0115(0.0036,0.0268)$ & 0.828 & 0.308 & -0.955 & 0.219 & 0.561 & -1.156 & 0.137 \\
\hline $\operatorname{ain} S$ & $0.1799(0.1488,0.2152)$ & $2.423(0.005)$ & $1.736(0.044)$ & 2.021 & 0.755 & $1.781(0.022)$ & -0.317 & 0.186 \\
\hline katA & $0.0402(0.0221,0.0661)$ & 0.701 & 0.410 & 0.530 & 0.021 & 0.363 & -0.244 & 0.131 \\
\hline pyrC & $0.0263(0.0136,0.0450)$ & 0.004 & -0.221 & -1.476 & 0.267 & 0.228 & -3.094 & 0.109 \\
\hline $\operatorname{lux} A$ & $0.0642(0.0525,0.0776)$ & $3.034(<0.001)$ & $1.706(0.020)$ & $2.890(<0.001)$ & $1.964(<0.001)$ & $2.979(<0.001)$ & $19.610(<0.001)$ & $0.207(<0.001)$ \\
\hline
\end{tabular}

${ }^{\mathrm{a}} K_{\mathrm{a}} / K_{\mathrm{s}}$ or $d_{\mathrm{N}} / d_{\mathrm{s}}$ : Ratio of the number of non-synonymous substitutions per non-synonymous site to the number of synonymous substitutions per synonymous site, calculated using the SLAC method (95\% confidence interval in parentheses).

bTajima's $D$ test statistic calculated using the nucleotide sequence and the total number of segregating sites $(S)$, total mutations $(\eta)$ or the aminoacid sequence and the total number of segregating sites $(A A)$.

${ }^{\text {c}}$ Statistical significance of test statistic (given in parentheses if $P<0.05$ ) was calculated from $10^{3}$ neutral coalescence simulations by using segregating sites with an intermediate level of recombination.

${ }^{\mathrm{d}} \mathrm{Fu}$ and Li's $D$ and $F$, and Fu's Fs test statistics were calculated by using total segregating sites.

epopulation growth test statistic of Ramos-Onsis and Rozas (Ramos-Onsins and Rozas, 2002); the test statistic distribution and statistical significance were calculated from $10^{3}$ neutral coalescence simulations by using segregating sites with an intermediate level of recombination. For the lux $A$ fragment, the test statistic is significantly larger than the distribution calculated by the coalescent at a level of $P<0.001$.

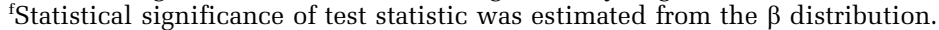


$P<0.001$ ) implying a relatively clonal population structure for the $45 \mathrm{~V}$. fischeri strains analyzed. For a concatenation of the six loci, $r / m$ was calculated as $1.46(95 \%$ confidence interval $=1.06-1.90)$; this value is most likely an underestimation of the summary statistic because the sequence collection itself represents a global sample that may include multiple local subpopulations. A value of 1.46 is typical of a free-living bacterial species (Vos and Didelot, 2009); both of these recombination metrics support the hypothesis that recombination has an important, yet moderate, role in the generation of diversity among $V$. fischeri.

\section{Phylogenetic reconstruction of concatenated loci and comparison with VfRep-PCR data}

To assess the evolutionary significance of the $V$. fischeri-specific VfRep-PCR fingerprinting data, a multi-locus sequence analysis approach was used to analyze the phylogenetic relationships among $V$. fischeri. Multi-locus analyses have the advantage of combining the weak phylogenetic signal present in multiple housekeeping (or other) loci into a significantly well-resolved reconstruction through the aggregation of phylogenetic signal using informative sites (Hanage et al., 2006). One important caveat to this approach is that loci with a relatively large number of polymorphisms have the potential to contribute a disproportionate amount of information to the final reconstruction. For the $V$. fischeri data analyzed above, single-locus analyses demonstrated that one of the locus fragments, $\operatorname{lux} A$, had both a disproportionate amount of variable sites and strong violations of neutral theory relative to the other five loci. Furthermore, the other bioluminescence-related locus, ainS, had similar, but slightly less serious, shortcomings (Table 1 and Supplementary Table S4). Therefore, phylogenetic reconstructions of a concatenation made with only the four 'housekeeping' loci (recAmdhkatApyrC) (Figure 2 and Supplementary Figure S2) were overlaid with VfRep-PCR type data (Supplementary Table S1).

For all reconstructions, E. scolopes symbionts formed a well-supported, but polyphyletic clade. Within this clade, VfRep-PCR type-I strains all formed a well-supported, monophyletic clade, 'Group-A', that was distal to all other $V$. fischeri taxa (Figure 2 and Supplementary Figure S2). VfRep-PCR type-II and III strains formed a well-supported, paraphyletic clade ('non-Group-A') containing the more distal Group-A clade. The Group-A clade contained not only contemporary symbionts isolated in 2005, but also two strains (ES12, ES213) isolated from the light organs of E. scolopes collected in Maunalua Bay nearly 20 years earlier (Lee, 1994).

Fitness assays of Group-A strains compared with other Hawaiian V. fischeri strains

In a previous study (Wollenberg and Ruby, 2009), MLST Group-A (that is, VfRep-PCR type-I) strains dominated the symbiont populations in the E. scolopes hosts collected from Maunalua Bay, Hawaii, in 2005. With the observations of the relative homogeneity of the Group-A $V$. fischeri strains, and their abundance relative to other Hawaiian $V$. fischeri strains, we hypothesized that these strains were more fit in both the free-living and the host-associated environments encountered in Maunalua Bay, Hawaii. To test these hypotheses, growth and survival assays were performed both in MB E. scolopes hosts and in fresh MB seawater.

Competition assays in juvenile MB hosts demonstrated that $V$. fischeri MLST Group-A strains showed a colonization advantage over the nonGroup-A strains (Figure 3). When different GroupA strains were individually competed against one of two different non-Group-A strains for $48 \mathrm{~h}$, the Group-A strains dominated the majority of competition experiments. In all experiments, Group-A strains were also found to be colonizing as an apparent monoculture in a disproportionately high percentage of juveniles after $48 \mathrm{~h}$ of competition. Variables such as time of initial inoculation, concentration of initial inoculant and strain identity did not affect these results qualitatively (Figure 3). Additionally, another non-Group-A strain, ES114, showed similar competition results against Group-A strains after both 3 and 18-h inoculation periods (data not shown).

One possible explanation for this observation of competitive advantage of Group-A strains over nonGroup-A strains in $\mathrm{MB}$ juveniles is an improved ability of the Group-A strains to initiate symbiosis with MB juveniles. To test this hypothesis, singlestrain colonization experiments of juvenile E. scolopes from MB were performed (Figure 4). The results of these experiments demonstrated that individual strains varied in their abundance in $\mathrm{MB}$ juveniles at $24 \mathrm{~h}, 48 \mathrm{~h}$ and $72 \mathrm{~h}$ after infection, and that there was no statistically significant difference between the mean abundance of Group-A strains and non-GroupA strains at these time points $\left(F_{1.4}=0.258, P=0.64\right)$. These data are not surprising, as the ability to discern patterns of difference in colonization fitness between strains often requires co-colonization experiments (Stabb and Ruby, 2003).

Incubation for 4 days in fresh, unfiltered $\mathrm{MB}$ seawater revealed significantly different growth and survival responses of Group-A $V$. fischeri relative to non-Group-A V. fischeri (Figure 5). Seawater growth/ survival data from filtered seawater showed no qualitative or quantitative difference between Group-A and non-Group-A V. fischeri strains. For all strains, abundance increased 2- to 10-fold for the first $12 \mathrm{~h}$, followed by a 10 -fold decrease by $96 \mathrm{~h}$. By contrast, in unfiltered seawater, Group-A strains showed a more rapid decrease in abundance after $12 \mathrm{~h}$ than non-Group-A V. fischeri strains. Results of a two-way, repeated-measures analysis of variance included a significant interaction term between Group identity and time $\left(F_{5,80}=10.368, P<0.001\right)$. 

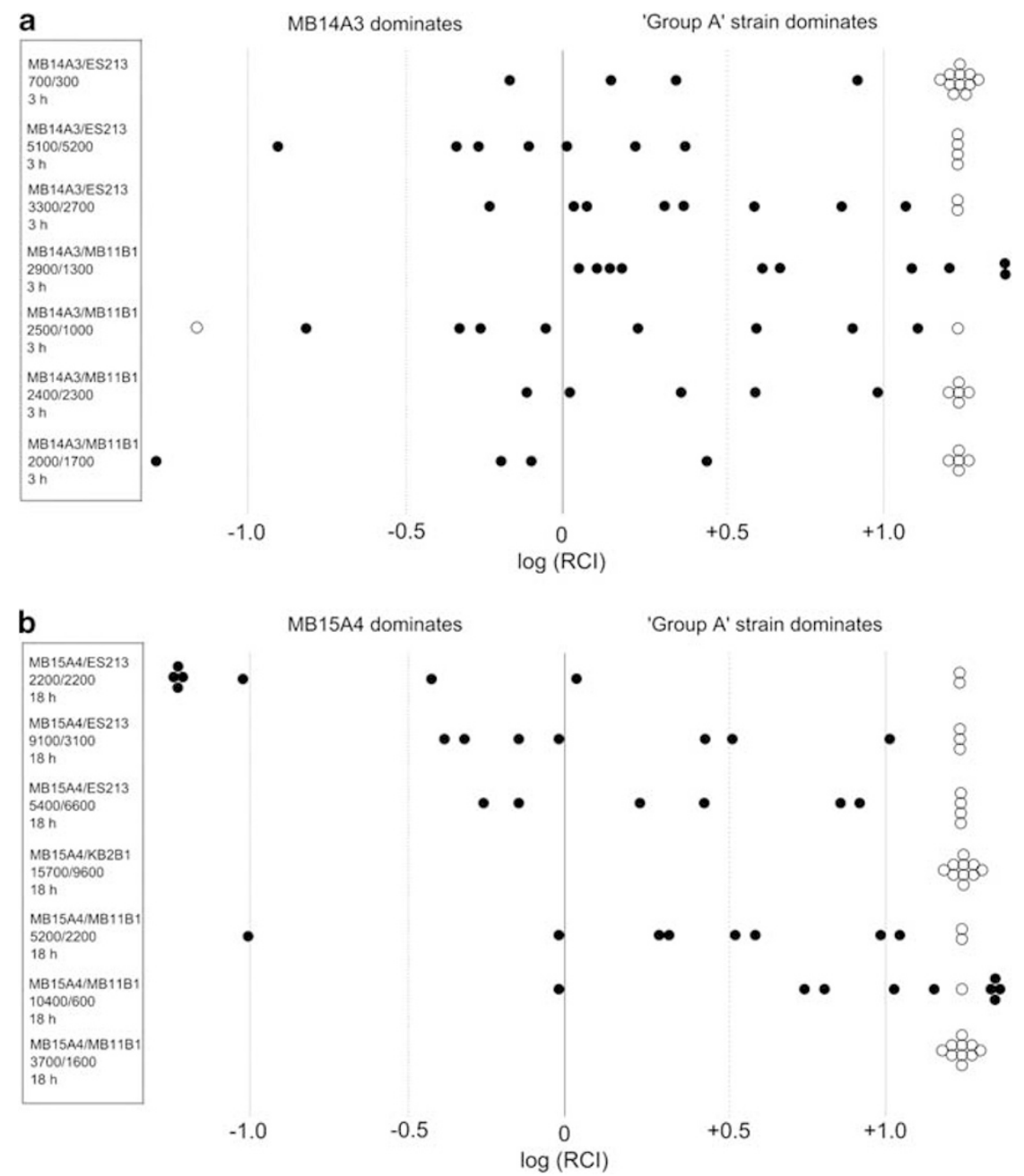

Figure 3 Dual-strain, juvenile E. scolopes experiments competing single $V$. fischeri 'Group-A' strains against either (a) MB14A3 or (b) MB15A4. In both graphs, each row represents a single experiment, summarized by the box on the left as follows: First line, strains' identities; second line, initial mean concentration of each strain in c.f.u./ml (the s.d. was less than $50 \%$ of this mean value for all experiments); third line, duration of inoculation. All experiments were concluded at $48 \mathrm{~h}$ after hatching and involved 9-15 juvenile squid born from mating adults collected from Maunalua Bay, Hawaii. The unfilled circles represent individual squid that were found to contain only one of the strains (that is, the other strain was below the limit of detection-approximately 500 times less abundant than the strain present). The unfilled circles are arbitrarily positioned at 1.25 and -1.25 units on each axis. Each filled circle represents an individual squid that contained both strains, and is positioned on the graph as the logarithm of the RCI (logRCI) for the two strains. Filled circles with a logRCI value greater than 1.25 or less than -1.25 are placed either to the right or to the left of these values (and unfilled circles), respectively, because of graphical limitations.

This test result was interpreted as follows: a significant difference exists between the mean abundance profile of the Group-A strains and the non-Group-A strains over the duration of the experiment. Qualitatively, this difference appears as a sharp decline after $48 \mathrm{~h}$ for the Group-A strains, compared with a slow decline for the other strains. In filtered $\mathrm{MB}$ seawater, results of two-way, repeated-measures analysis of variance included a non-significant interaction term between Group identity and time $\left(F_{5,80}=1.497, P=0.20\right)$.

\section{Discussion}

This and another recent study (Wollenberg and Ruby, 2009) have focused on the population biology of symbiotic $V$. fischeri from a particular ecological and geographical setting: the Hawaiian bobtail squid E. scolopes from the island of Oahu. In the previous study, phenotypic assays and molecular fingerprinting revealed that the symbiotic populations of adult E. scolopes light organs are oligoclonal. Here, we advance these ecological observations with a study of these light-organ symbionts by using evolutionary inference and experimental fitness assays. We show here that a monophyletic group of $V$. fischeri (Group-A) is found disproportionately in hosts from Maunalua Bay, Oahu, Hawaii (MB). This new observation led to an additional question: Do members of Group-A, when compared with other symbiotic $V$. fischeri not in this group, demonstrate increased fitness in the Maunalua Bay environments? 
Experiments using the offspring of $\mathrm{MB}$ squid suggest that $V$. fischeri strains from Group-A are more fit in the MB E. scolopes light-organ niche than the non-Group-A strains (Figure 3). These results demonstrate the competitive advantage of a collection of closely related, symbiotic $V$. fischeri at a previously unstudied scale: a local, intra-island distance $(<20 \mathrm{~km})$ between bays on Oahu, Hawaii. In the past, $V$. fischeri strains from squid populations separated by larger geographic distances $(>2000 \mathrm{~km}$ ) have been shown to exhibit a competitive advantage colonizing juvenile hosts from the strain's local environment (Nishiguchi et al., 1998; Nishiguchi, 2002). Although the molecular determinants of host specificity are beginning to be reported in V. fischeri (Mandel, 2010), and there exists evidence to suggest that the response regulator $\operatorname{rsc} S$

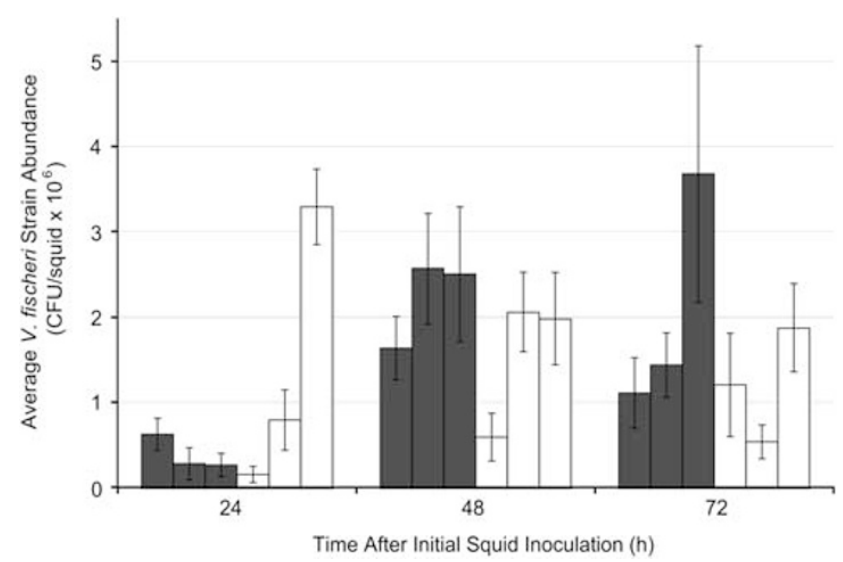

Figure 4 Single-strain $V$. fischeri inoculations of juvenile squid demonstrated no clear difference between the levels of symbiosis initiation by Group-A and non-Group-A V. fischeri. Each bar represents the mean c.f.u./squid of 10-15 squid inoculated with a single isolate of $V$. fischeri and sacrificed at 24,48 or $72 \mathrm{~h}$ after inoculation. The error bars represent $95 \%$ confidence intervals. The histogram bars relate to $V$. fischeri strains as follows: Non-'Group-A' $V$. fischeri strains are represented by the three black bars (left, ES114; center, MB14A3; right, MB15A4), whereas 'Group-A' V. fischeri strains are represented by the three white bars (left, ES213; center, MB11B1; right, KB2B1). may have some role in determining the host range (that is, squid or fish) of $V$. fischeri symbionts (Mandel et al., 2009), to date there has been no work defining what molecule(s) might mediate the competitive advantage of one strain of $V$. fischeri over another in the same host species at such a local geographic scale.

The Group-A and non-Group-A strains showed equivalent growth and persistence in $0.2-\mu \mathrm{m}$-filtered MB seawater (Figure 5a), suggesting that the innate ability of different $V$. fischeri strains to survive starvation is comparable. As shown previously (Lee and Ruby, 1994), incubation of $V$. fischeri cells in unfiltered, fresh seawater led to decreased (culturable) cell counts (c.f.u.s) after the first 12-24h of initial growth. Interestingly, in contrast to their competitive dominance in juvenile E. scolopes, the Group-A strains were at a statistically significant disadvantage in persisting in fresh, unfiltered $\mathrm{MB}$ seawater after $12 \mathrm{~h}$ when compared with the nonGroup-A strains (Figure 5b). Taken together, results from these studies suggest a link between (biotic or abiotic) particulate factors in Maunalua Bay seawater and the abundance and survival of $V$. fischeri cells in the planktonic environment.

How might future studies make sense of both the phylogenetic and the contrasting growth/survival characteristics of $V$. fischeri strains from Oahu? One attractive conceptual framework for the evolution of the Group-A strains involves the hypothesis that host population biogeography has driven local symbiont evolution-in other words, $V$. fischeri evolution in response to selection by the local host population. Another (possibly mutually exclusive) working hypothesis consistent with these results is that the evolution of the Group-A strains has involved a more general trade-off between growth in the light-organ environment and survival in the seawater environment.

Limited evidence exists in support of the former hypothesis that host biogeography drives microbial symbiont evolution at an intra-island scale. First, molecular and phenotypic data suggest that the
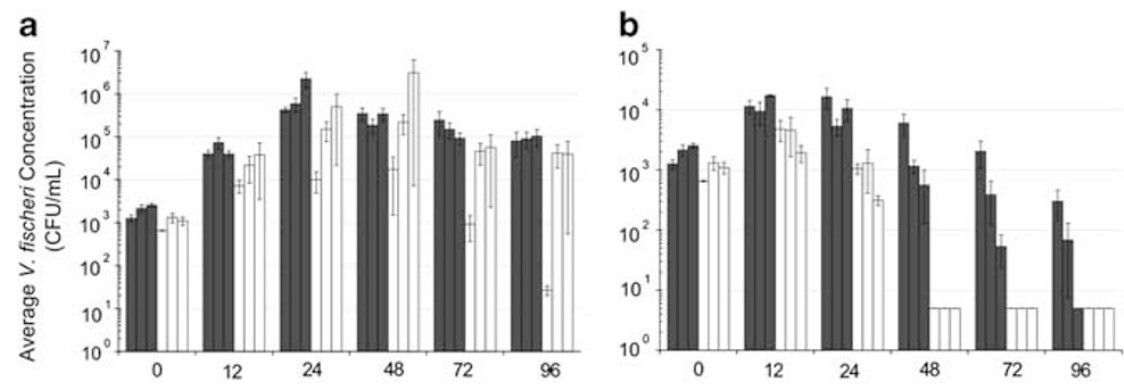

Figure 5 Single-strain $V$. fischeri growth experiments in microcosms filled with either (a) 0.22 - $\mu$ m-filtered or (b) unfiltered fresh seawater from Maunalua Bay, Hawaii. In both graphs, each bar represents the mean c.f.u./ml of three biological replicates of a single strain, with error bars indicating the s.e.m. The histogram bars relate to $V$. fischeri strains as follows: Non-'Group-A' $V$. fischeri are represented by the three black bars at each time point (left, ES114; center, MB14A3; right, MB15A4), whereas 'Group-A' V. fischeri are represented by the white bars at each time point (left, ES213; center, MB11B1; right, KB2B1). In the unfiltered seawater data (b), the mean abundance values that are indicated at 3 c.f.u. $\mathrm{ml}^{-1}$ for the 48 - to 96 -h time points were below this limit of detection. 
E. scolopes population on Oahu shows a local structure between Maunalua and Kaneohe Bays (Kimbell et al., 2002). Second, the total number of $V$. fischeri cells in the planktonic environment has been estimated to be several (Jones et al., 2007) to many (Lee and Ruby, 1995) orders of magnitude lower than in the host light organs, suggesting that the seawater niche may have a relatively minor role in $V$. fischeri evolution. Finally, recent studies of $V$. fischeri populations in E. scolopes have demonstrated that the light-organ environment can show strong selective pressure on the physiology of $V$. fischeri (Schuster et al., 2010). Taken together, this evidence suggests many key features related to the host E. scolopes (that is, local population structure; strong, selective environment; high symbiont population density) that might potentiate the evolution of local $V$. fischeri strains. Studies of other horizontal, 'binary' symbioses have argued that local host populations may have some influence on the local symbiont population structure and evolution (see, for example, Santos et al., 2003; Sicard et al., 2004; Cafaro et al., 2010; Maneesakorn et al., 2011), although other arguments also exist (for example, Martínez-Romero, 2009; Mueller et al., 2010), even within the squid/Vibrio symbiosis (Nishiguchi et al., 1998; Dunlap et al., 2007). For instance, using different phylogenetic markers and approaches, the latter two citations arrive at opposing conclusions concerning the evidence for parallel cladogenesis between species of squid and their light-organ symbionts.

Alternatively, our results might best be addressed by the second hypothesis that general trade-offs exist between $V$. fischeri growth/survival in the two environments studied (seawater and the squid host). Phenotypic and/or genotypic trade-offs have been hypothesized to explain the life-history differences observed among or within evolutionary lineage(s) in two or more ecological niches (Stearns, 1992). Contemporary microbial ecology research has sometimes focused on the trade-offs microorganisms experience when grown in environments either sufficient or lacking in metabolites (Velicer and Lenski, 1999). In the case of $V$. fischeri, the E. scolopes light organ is thought to provide a dynamic, nutrient-rich habitat (Ruby et al., 2005; Wier et al., 2010) in contrast to the relatively oligotrophic planktonic environment (Giovannoni and Stingl, 2007). In ecological terms, then, symbiotic $V$. fischeri around Oahu might inhabit a heterogeneous environment composed of two physiologically distinct habitats: seawater and the host light organ.

Additional experiments, both in juvenile E. scolopes and in the Hawaiian planktonic environment, will be needed to evaluate the relative robustness of the local host specificity and the host/seawater trade-off hypotheses. For instance, experimental evolution from a single parental genotype has provided a highly rigorous approach to measuring differences in bacterial traits associated with environmental trade-offs (Bennett and Lenski, 2007). Because experimental evolution can be performed in the squid host (Schuster et al., 2010), similar studies in E. scolopes from a certain Oahu population (for example, MB), using a known squid symbiont (for example, the non-Group-A strain $V$. fischeri ES114), could examine trade-offs between the light-organ and seawater habitats. Similarly, it will be important to determine whether the planktonic habitat represents an important non-symbiotic reservoir for $V$. fischeri proliferation, or simply a carbon-poor environment that must be survived on the way to a more nutritive niche (for example, the squid light organ).

In summary, the evolutionary concepts of tradeoffs and local-host/symbiont co-evolution and specificity will provide useful frameworks for further study of sympatric $V$. fischeri collected from Oahu, Hawaii. With the help of additional observational and experimental work both in the host and in Hawaiian seawater, the squid/Vibrio association will continue to be a model system in which the study of an inter-kingdom mutualism enriches knowledge of bacterial ecology and evolution.

\section{Acknowledgements}

We thank the members of the Ruby and McFall-Ngai labs for discussing this research and the resulting manuscript. We are also grateful for the statistical advice of $C$ Ané and the critical editing of $\mathrm{H}$ Goodrich-Blair. R Gates provided support and laboratory resources at the Hawaiian Institute for Marine Biology (HIMB) during collection. MSW thanks both $\mathrm{H}$ and $\mathrm{T}$ Putnam for help in the logistical aspects of the Hawaii fieldwork. MSW was supported by NIH Molecular Biosciences (5T32GM007215-35) and NIH Microbes in Health and Disease (2T32AI055397-07) Training Grants through University of Wisconsin-Madison. Research support was provided by NSF IOS 0841507 to M McFall-Ngai and EGR, and NIH R01 RR12294 to EGR and M McFall-Ngai.

\section{References}

Bennett AF, Lenski RE. (2007). An experimental test of evolutionary trade-offs during temperature adaptation. Proc Natl Acad Sci USA 104: 8649-8654.

Boettcher KJ, Ruby EG. (1990). Depressed light emission by symbiotic Vibrio fischeri of the sepiolid squid Euprymna scolopes. J Bacteriol 172: 3701-3706.

Bose JL, Wollenberg MS, Colton DM, Mandel MJ, Septer AN, Dunn AK et al. (2011). Contribution of rapid evolution of the luxR-luxI intergenic region to the diverse bioluminescence output of Vibrio fischeri strains isolated from different environments. Appl Environ Microbiol 77: 2445-2457.

Cafaro MJ, Poulsen M, Little AE, Price SL, Gerardo NM, Wong B et al. (2010). Specificity in the symbiotic association between fungus-growing ants and protective Pseudonocardia bacteria. Proc Biol Sci 278: 1814-1822. 
Didelot X, Falush D. (2007). Inference of bacterial microevolution using multilocus sequence data. Genetics 175: 1251-1266.

Dunlap PV, Ast JC, Kimura H, Fukui A, Yoshino T, Endo H. (2007). Phylogenetic analysis of host-symbiont specificity and codivergence in bioluminescent symbioses. Cladistics 23: 507-532.

Dykhuizen DE, Green L. (1991). Recombination in Escherichia coli and the definition of biological species. J Bacteriol 173: 7257-7268.

Edgar RC. (2004). MUSCLE: multiple sequence alignment with high accuracy and high throughput. Nucleic Acids Res 32: 1792-1797.

Gelman A, Rubin DB. (1992). Inference from iterative simulation using multiple sequences. Stat Sci 7: 457-472.

Giovannoni S, Stingl U. (2007). The importance of culturing bacterioplankton in the 'omics' age. Nat Rev Microbiol 5: 820-826.

Gouy M, Guindon S, Gascuel O. (2010). SeaView version 4: a multiplatform graphical user interface for sequence alignment and phylogenetic tree building. $\mathrm{Mol}$ Biol Evol 27: 221-224.

Hanage WP, Fraser C, Spratt BG. (2006). Sequences, sequence clusters and bacterial species. Philos Trans $R$ Soc Lond B Biol Sci 361: 1917-1927.

Holland BR, Huber KT, Moulton V, Lockhart PJ. (2004). Using consensus networks to visualize contradictory evidence for species phylogeny. Mol Biol Evol 21: 1459-1461.

Huson DH, Bryant D. (2006). Application of phylogenetic networks in evolutionary studies. Mol Biol Evol 23: 254-267.

Jones BW, Maruyama A, Ouverney CC, Nishiguchi MK. (2007). Spatial and temporal distribution of the Vibrionaceae in coastal waters of Hawaii, Australia, and France. Microb Ecol 54: 314-323.

Kimbell JR, McFall-Ngai MJ, Roderick GK. (2002). Two genetically distinct populations of bobtail squid, Euprymna scolopes, exist on the island of Oahu. Pac Science 56: 347-355.

Kimura M. (1983). The Neutral Theory of Molecular Evolution. Cambridge University Press: Cambridge.

Lee K, Ruby EG. (1995). Symbiotic role of the viable but nonculturable state of Vibrio fischeri in Hawaiian coastal seawater. Appl Environ Microbiol 61: 278-283.

Lee KH, Ruby EG. (1992). Detection of the light organ symbiont, Vibrio fischeri, in Hawaiian seawater by using lux gene probes. Appl Environ Microbiol 58: 942-947.

Lee KH. (1994). Ecology of Vibrio fischeri, the Light Organ Symbiont of the Hawaiian Sepiolid Squid Euprymna scolopes. University of Southern California: Los Angeles.

Lee KH, Ruby EG. (1994). Effect of the squid host on the abundance and distribution of symbiotic Vibrio fischeri in nature. Appl Environ Microbiol 60: 1565-1571.

Mandel MJ, Wollenberg MS, Stabb EV, Visick KL, Ruby EG. (2009). A single regulatory gene is sufficient to alter bacterial host range. Nature 458: 215-218.

Mandel MJ. (2010). Models and approaches to dissect hostsymbiont specificity. Trends Microbiol 18: 504-511.

Maneesakorn P, An R, Daneshvar H, Taylor K, Bai X, Adams BJ et al. (2011). Phylogenetic and cophylogenetic relationships of entomopathogenic nematodes (Heterorhabditis: Rhabditida) and their symbiotic bacteria (Photorhabdus: Enterobacteriaceae). Mol Phylogenet Evol 59: 271-280.
Martínez-Romero E. (2009). Coevolution in Rhizobiumlegume symbiosis? DNA Cell Biol 28: 361-370.

McFall-Ngai MJ, Ruby EG. (1991). Symbiont recognition and subsequent morphogenesis as early events in an animal-bacterial mutualism. Science 254: 1491-1494.

Mueller UG, Ishak H, Lee JC, Sen R, Gutell RR. (2010). Placement of attine ant-associated Pseudonocardia in a global Pseudonocardia phylogeny (Pseudonocardiaceae, Actinomycetales): a test of two symbiont-association models. Antonie Van Leeuwenhoek 98: 195-212.

Nishiguchi MK, Ruby EG, McFall-Ngai MJ. (1998). Competitive dominance among strains of luminous bacteria provides an unusual form of evidence for parallel evolution in Sepiolid squid-Vibrio symbioses. Appl Environ Microbiol 64: 3209-3213.

Nishiguchi MK. (2002). Host-symbiont recognition in the environmentally transmitted sepiolid squid-Vibrio mutualism. Microb Ecol 44: 10-18.

Ramos-Onsins SE, Rozas J. (2002). Statistical properties of new neutrality tests against population growth. Mol Biol Evol 19: 2092-2100.

R_Development_Core_Team (2010). R: a Language and Environment for Statistical Computing. R Foundation for Statistical Computing: Vienna, Austria.

Ruby EG, Urbanowski M, Campbell J, Dunn A, Faini M, Gunsalus $\mathrm{R}$ et al. (2005). Complete genome sequence of Vibrio fischeri: a symbiotic bacterium with pathogenic congeners. Proc Natl Acad Sci USA 102: 3004-3009.

Santos SR, Gutiérrez-Rodríguez C, Lasker HR, Coffroth MA. (2003). Symbiodinium sp. associations in the gorgonian Pseudopterogorgia elisabethae in the Bahamas: high levels of genetic variability and population structure in symbiotic dinoflagellates. Marine Biol 143: 111-120.

Schuster BM, Perry LA, Cooper VS, Whistler CA. (2010). Breaking the language barrier: experimental evolution of non-native Vibrio fischeri in squid tailors luminescence to the host. Symbiosis 51: 85-96.

Sicard M, Ferdy JB, Pages S, Le Brun N, Godelle B, Boemare $\mathrm{N}$ et al. (2004). When mutualists are pathogens: an experimental study of the symbioses between Steinernema (entomopathogenic nematodes) and Xenorhabdus (bacteria). J Evol Biol 17: 985-993.

Simonsen KL, Churchill GA, Aquadro CF. (1995). Properties of statistical tests of neutrality for DNA polymorphism data. Genetics 141: 413-429.

Stabb EV, Ruby EG. (2003). Contribution of pilA to competitive colonization of the squid Euprymna scolopes by Vibrio fischeri. Appl Environ Microbiol 69: 820-826.

Stabb EV, Millikan DS. (2009). Is the $V$. fischeri-E. scolopes symbiosis a defensive mutualism?. In: White JF, Torres MS (eds) Defensive Mutualism in Microbial Symbiosis. CRC Press: New York City, pp 85-98.

Stearns SC. (1992). The Evolution of Life Histories. Oxford University Press: New York, NY.

Tajima F. (1989). The effect of change in population size on DNA polymorphism. Genetics 123: 597-601.

Velicer GJ, Lenski RE. (1999). Evolutionary trade-offs under conditions of resource abundance and scarcity: experiments with bacteria. Ecology 80: 1168-1179.

Visick KL, Ruby EG. (2006). Vibrio fischeri and its host: it takes two to tango. Curr Opin Microbiol 9: 632-638.

Vos M, Velicer GJ. (2006). Genetic population structure of the soil bacterium Myxococcus xanthus at the centimeter scale. Appl Environ Microbiol 72: 3615-3625. 
Vos M, Didelot X. (2009). A comparison of homologous recombination rates in bacteria and archaea. ISME J 3: 199-208.

Wei SL, Young RE. (1989). Development of symbiotic bioluminescence in a nearshore cephalopod, Euprymna scolopes. Marine Biol 103: 541-546.

Wier AM, Nyholm SV, Mandel MJ, Massengo-Tiasse RP, Schaefer AL, Koroleva I et al. (2010). Transcriptional patterns in both host and bacterium underlie a daily rhythm of anatomical and metabolic change in a beneficial symbiosis. Proc Natl Acad Sci USA 107: 2259-2264.

Wollenberg MS, Ruby EG. (2009). Population structure of Vibrio fischeri within the light organs of Euprymna scolopes squid from two Oahu (Hawaii) populations. Appl Environ Microbiol 75: 193-202.

Supplementary Information accompanies the paper on The ISME Journal website (http://www.nature.com/ismej) 